\title{
Comparison of Pre and Post-operative Hearing Results in Canal Wall Down Mastoidectomy with Type III Tympanoplasty
}

\author{
Shrestha BL,' Bhusal CL,' Bhattarai $\mathrm{H}^{1}$ \\ 'Department of Otorhinolaryngology and Head and Neck Surgery, TUTH, Kathmandu, Nepal
}

\begin{abstract}
This study was done to compare the pre and post-operative hearing results in patients undergoing canal wall down mastoidectomy with classical type III tympanoplasty using temporalis fascia alone.

Patients of $\geq 5$ years age with the diagnosis of Chronic otitis media (squamous) with conductive or mixed hearing loss, needing canal wall down mastoidectomy and with intact and mobile stapes suprastructure at surgery who underwent classical type III tympanoplasty were included in the study. The pre and post-operative PTA was performed and evaluated. The post-operative hearing was assessed in terms of average ABG and size of ABG closure.
\end{abstract}

Mean pre and post-operative air bone gap in classical type III tympanoplasty were $37.8 \mathrm{~dB}$ and 29.8 $\mathrm{dB}$ respectively and these differences were statistically significant. The postoperative PTA-ABG ranged from 15-61.2 dB.

Hearing results after type III tympanoplasty varied widely showing statistically significant improvement in mean post-operative PTA-ABG but there was a great variation.

Key words: air bone gap, chronic otitis media, mastoidectomy, tympanoplasty

\section{INTRODUCTION}

Chronic otitis media (COM) is a chronic inflammatory disease of the middle ear and mastoid that often results in partial or total loss of the tympanic membrane (TM) and ossicles, leading to conductive hearing loss that can range in severity up to $60-70 \mathrm{~dB} .^{1}$

COM is a common condition, affecting $0.5-30 \%$ of any community. Therefore, a conservative estimate of the number of people in the world suffering from COM is over 20 million. $^{2}$ Among which the prevalence of squamous type of COM is $3.5 \%$ in Nepal. ${ }^{3}$

The goal of tympanoplasty is to restore sound pressure transformation at the oval window by coupling an intact tympanic membrane with a mobile stapes footplate via an intact or reconstructed ossicular chain and to provide sound protection for the round window membrane by a closed, air containing, mucosa lined

\footnotetext{
Correspondence:

Dr. Bikash Lal Shrestha

Department of Otorhinolaryngology and Head and Neck Surgery, TU Teaching Hospital, Kathmandu, Nepal.

Email: bikash001@hotmail.com
} 
Shrestha et al. Comparison of Pre and Post-operative Hearing Rearing Results in Canal Wall...

middle ear. ${ }^{4}$ The modern era of tympanoplasty was ushered in by Wullstein and Zollner. Wullstein classified the operations as types I through $V$.

The type III tympanoplasty is a well established technique for middle ear reconstruction when tympanic membrane, malleus and incus are missing or diseased, as is often seen in chronic otitis media (COM). ${ }^{4}$ In classical type III tympanoplasty or myringostapediopexy, disease is removed from tympanomastoid compartment and advancement of the tympanic membrane(TM) or placement of tissue graft is done on top of the stapes capitulum. The air-bone gap ( $A B G$ ) range from 10-60 $\mathrm{dB}$ after this procedure.

\section{MATERIAL AND METHODS}

A Prospective study performed in Ganesh Man Singh Memorial Academy of ENT and Head and Neck Studies, Tribhuvan University Teaching Hospital, Institute of Medicine, Maharajgunj, Kathmandu, Nepal from October 2006 to April 2008 and patients who were of $\geq 5$ years, both gender, intact and mobile stapes suprastructure at CWD tympanomastoidectomy surgery for COM squamous type were included.

The Pure Tone Audiometry test performed within seven days prior to the operation by Hughson and Westlake method. The test was performed through Air Conduction and Bone Conduction mode. The Air Conduction threshold and the Bone Conduction threshold averages were calculated by taking the averages of 500, 1000, 2000 and $4000 \mathrm{~Hz}$ frequencies. The ABG was calculated by taking differences between Air conduction and Bone Conduction threshold. The Air and Bone conduction threshold was recorded both pre and post-operatively. The postoperative air bone gap ( $A B G$ ) closure was calculated by taking the difference between the preoperative $A B G$ and the postoperative $A B G$ of the average frequencies of 500, 1000, 2000 and 4000 $\mathrm{Hz}$. The audiometry results were reported according to American Academy of Otolaryngology-Head and Neck Surgery guidelines, except for thresholds at $3 \mathrm{kHz}$, which were substituted in all cases with thresholds at $4 \mathrm{kHz}{ }^{5}$

For classical type III tympanoplasty, a temporalis fascia graft was used to bridge the middle ear air space and placed in contact with the stapes head. The graft was then draped over the facial ridge.

The follow up of patients were performed at or after $10^{\text {th }}$ week postoperatively. During follow up period, ears with minimal discharge from the mastoid cavity but with healed middle ear were subjected to pure tone audiometry (PTA) assessment.

The results were analyzed in terms of average postoperative $A B G$ and $A B G$ closure. $P$ value was calculated using the independent samples test and $P$ value of less than or equal to 0.05 was taken as significant. The data analysis was performed with the help of statistical software for social sciences (SPSS) version 11.5 for windows.

\section{RESULTS}

The total number of patients enrolled during the study period were 41 (53.2\%) cases among these patients, $39(95.1 \%)$ came for follow up and $2(4.9 \%)$ patients were lost in the follow up. Among the cases from adequate follow up, $1(2.4 \%)$ case was excluded from the study because of graft failure (Table 1 ).

The patients were divided into different groups of age. Patients of $\leq 10$ years were $4(10.5 \%), 11-20$ years were $17(44.7 \%), 21-30$ years were $11(28.9 \%), 31$ 40 years were $3(7.9 \%)$ and $>40$ years were $3(7.9 \%)$. The average age was $21.03 \pm 6.07$ years (Table 2 ).

The Post-operative ABG at frequencies $500 \mathrm{~Hz}, 1000$ $\mathrm{Hz}, 2000 \mathrm{~Hz}$, and $4000 \mathrm{~Hz}$ were found to be 37.9 $\mathrm{dB}, 30.1 \mathrm{~dB}, 22.0 \mathrm{~dB}$ and $29.2 \mathrm{~dB}$ respectively as compared to pre-operative $A B G$ of $46.6 \mathrm{~dB}, 39.7 \mathrm{~dB}$, $29.9 \mathrm{~dB}$ and $35.0 \mathrm{~dB}$ in these respective frequencies. These differences were statistically significant except at $4000 \mathrm{~Hz}$.

The four frequency average pre-operative $A B G$ which was $37.8 \mathrm{~dB}$ was reduced to 29.8 post-operatively with a net gain of $8.0 \mathrm{~dB}$. This observed difference was found to be statistically highly significant with $p$ value of $<0.001$. It was observed that the $A B G$ was the smallest at $2000 \mathrm{~Hz}$ as compared with other frequencies in both pre and post-operative audiograms.

The $A B G$ closure was divided into different categories like 0-5dB, 0-10 dB, 0-20 dB, 0-30 dB and $>30 \mathrm{~dB}$. It was noted that $7(18.4 \%)$ cases fell within $0-5 \mathrm{~dB}$ and $23(60.5 \%)$ within 0-20 dB (Table 4$)$.

\section{DISCUSSION}

The objectives of this study were to compare the pre and post-operative hearing results in terms of average $A B G$ and the size of $A B G$ closure in patients undergoing CWD mastoidectomy with classical type III tympanoplasty using temporalis fascia.

In our study the average age of the patients in was 21.03 , the range being 5 to 55 years. But most of the similar studies done in the literature have included patients in their fourth or fifth decade and the range also shows patients of younger and older age groups. ${ }^{6-9}$ This variation may be attributed to the socio-cultural context that in our country older adults are a bit hesitant to undergo surgical treatment as compared to patients of younger age. 
Shrestha et al. Comparison of Pre and Post-operative Hearing Rearing Results in Canal Wall...

Table 1. Total distribution of patients $(n=41)$

\begin{tabular}{llccc}
\hline Groups & Total cases & Included & Excluded & Lost follow up \\
\hline CWD mastoidectomy and classical type III tympanoplasty & $41(100 \%)$ & $38(92.7 \%)$ & $1(2.4 \%)$ & $2(4.9 \%)$ \\
\hline
\end{tabular}

Table 2. Age distribution of patients $(n=38)$

\begin{tabular}{lc}
\hline Age (years) & $\begin{array}{l}\text { CWD mastoidectomy and classical } \\
\text { type III tympanoplasty }\end{array}$ \\
\hline$\leq 10$ & $4(10.5 \%)$ \\
$11-20$ & $17(44.7 \%)$ \\
$21-30$ & $11(28.9 \%)$ \\
$31-40$ & $3(7.9 \%)$ \\
$>40$ & $3(7.9 \%)$ \\
\hline
\end{tabular}

We reported only short-term hearing results, because the long-term success of any ossicular repair is largely dependent on factors outside the control of the surgeon, i.e. patient follow-up rates; eustachian tube function; middle-ear stability; and the condition of the mucosa. The short-term results are hence a more accurate reflection of the actual reconstructive procedures. In each case, air-bone gaps were calculated using air conduction and bone-conduction thresholds at frequencies 500, 1000, 2000 and $4000 \mathrm{~Hz}$.

Different methods have been used by different authors to report the pure tone audiometric post-operative hearing results in middle ear surgery in the literature. Among these $A B G$ closure, the post operative $A B G$ presented in $10 \mathrm{~dB}$ bins and air conduction threshold gain are commonly reported indicators of tympanoplasty outcome. We had applied average PTA-ABG and size of the $A B G$ closure for audiological assessment. Air Bone gap of the average of four frequencies 500, 1000, 2000 and $4000 \mathrm{~Hz}$ were used for analysis. For calculation of the size of the post-operative PTA- ABG closure ABGs were divided into different bins of $0-5 \mathrm{~dB}, 0-10 \mathrm{~dB}, 0-20$ $\mathrm{dB}, 0-30 \mathrm{~dB},>30 \mathrm{~dB}$.

In our study, the post-operative PTA-ABG showed statistically significant improvement as compared to pre operative PTA-ABG. Patients had average PTA-ABG of $37.8 \mathrm{~dB}$ pre-operatively and $29.8 \mathrm{~dB}$ post-operatively with a net gain of $8 \mathrm{~dB}$. The difference between the pre and post-operative PTA-ABG results was statistically significant $(P<.001)$. Our results are similar to the results of myringostapediopexy of Cheang et al. ${ }^{10}$ In his patients $(n=25)$ PTA-ABG pre- and post operatively were $28.25 \mathrm{~dB}$ and $24.7 \mathrm{~dB}$. However our results are different from those of Dawes PJD. ${ }^{7}$ He reports the average PTA- ABG using frequencies .5, 1, and $2 \mathrm{kHz}$ of naturally formed myringostapediopexy $(n=15)$ and myringostapediopexy formed after canal wall down mastoidectomy $(n=35)$ to be 17.7 and $19.0 \mathrm{~dB}$, the range being 5-41.7 and 6.7-38.4 dB respectively. Depending upon a number of factors it is generally found that the success of myringostapediopexy is rather variable, the post operative PTA-ABG being anywhere between 10 and $60 \mathrm{~dB} .{ }^{1}$ Merchant et al report that type

Table 3. The pre and post-operative PTA-ABG evaluation of patients with CWD mastoidectomy and classical type III tympanoplasty $(n=38)$

\begin{tabular}{|c|c|c|c|c|c|c|c|}
\hline Parameter & Group & $\mathbf{n}$ & Mean & SD & Min. & Max. & $P$ value \\
\hline \multirow{10}{*}{$\begin{array}{l}\text { CWD mastoidectomy } \\
\text { and classical type III } \\
\text { tympanoplasty }\end{array}$} & Pre-operative ABG $500 \mathrm{~Hz}$ & \multirow{10}{*}{38} & 46.6 & 14.15 & 15 & 75 & \multirow{2}{*}{$<0.001$} \\
\hline & Post-operative ABG $500 \mathrm{~Hz}$ & & 37.9 & 13.74 & 15 & 75 & \\
\hline & Pre-operative ABG $1000 \mathrm{~Hz}$ & & 39.7 & 15.44 & 10 & 65 & \multirow{2}{*}{0.001} \\
\hline & Post-operative ABG $1000 \mathrm{~Hz}$ & & 30.1 & 14.12 & 10 & 65 & \\
\hline & Pre-operative ABG $2000 \mathrm{~Hz}$ & & 29.9 & 14.31 & 5 & 60 & \multirow{2}{*}{0.003} \\
\hline & Post-operative ABG $2000 \mathrm{~Hz}$ & & 22 & 12 & 5 & 55 & \\
\hline & Pre-operative ABG $4000 \mathrm{~Hz}$ & & 35 & 16.36 & 5 & 70 & \multirow{2}{*}{0.062} \\
\hline & Post-operative ABG $4000 \mathrm{~Hz}$ & & 29.2 & 14.73 & 10 & 80 & \\
\hline & Pre-operative ABG Average & & 37.8 & 12.96 & 13.7 & 60 & \multirow{2}{*}{$<0.001$} \\
\hline & Post-operative ABG Average & & 29.8 & 10.72 & 15 & 61.2 & \\
\hline
\end{tabular}

Table 4. ABG closure in classical type III tympanoplasty $(\mathbf{n}=\mathbf{3 8})$

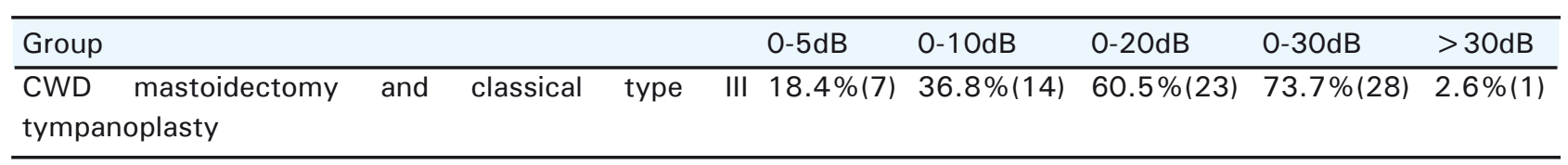


Shrestha et al. Comparison of Pre and Post-operative Hearing Rearing Results in Canal Wall...

III stapes collumella tympanoplasty in patients with mobile stapes and aerated middle ear result in mean $A B G$ of $15-30 \mathrm{~dB}$ but large $A B G$ of $30-70$ are found in ears with fixed stapes and nonaereated middle ear. ${ }^{9}$ As far as myringostapediopexy is concerned the results are unpredictable. Frequently the graft lateralizes, quite often atelectasis of the new drum occurs with adhesions in the middle ear and obliteration of the middle ear cleft. Retraction pockets are also not uncommon. ${ }^{11}$

While analyzing the frequency wise post-operative average PTA-ABG, it was seen that $A B G$ was the smallest at $2000 \mathrm{~Hz}$ as compared with other frequencies. Similar findings were also noted by Merchant SN et al in 2003.9 They explain that a combination of two factors are responsible for smaller ABG at $2000 \mathrm{~Hz}$. Bone-conduction threshold are not an exact measure of cochlear function and can be influenced by pathological condition of middle ear; the Carhart's notch phenomenon in otosclerosis is an example. Similarly, there is no clear explanation for the cause of the air conduction thresholds showing to be the lowest at $2000 \mathrm{~Hz}$ however; it could have resulted from resonances generated in the mastoid cavity and ear canal.

It was noted that $28(73.7 \%)$ cases in our study fell within $30 \mathrm{~dB}$ ABG closure. Our result compare well with myringostapediopexy as a tympanoplasty procedure in canal wall down mastoid surgery of Cheang et al ( $n=22$ ) who reports PTA-ABG closure within $30 \mathrm{~dB}$ in $61.9 \%$ of his such cases. ${ }^{10}$ Our results however are different from those of natural myringostapediopexy $(n=15)$ and surgical canal down myringostapediopexy $(n=35)$ of Dawes who reports PTA ABG closure within $30 \mathrm{~dB}$ in $87.0 \%$ and $90.0 \%$ of his cases in these two groups respectively. Moustafa and Khalifa observed hearing results of simple myringostapediopexy technique in $(n=145)$ and showed that only $10 \%$ of these cases achieved PTA-ABG closure of less than 30 dB. ${ }^{11,12}$

Functional results are often influenced by wide variability in the surgical techniques employed, in the criteria used to evaluate hearing results and a number of other anatomical, physiological and pathological events that occur post-operatively in the middle ear as mentioned above.

\section{CONCLUSION}

The results demonstrate that mean pre and postoperative air bone gap were $37.8 \mathrm{~dB}$ and $29.8 \mathrm{~dB}$ respectively with a net gain of $8 \mathrm{~dB}$. These differences were statistically significant. Similarly, the postoperative PTA-ABG ranged from 15-61.2 dB, suggesting the possibility of effect of multiple other factors. Therefore, when indicated CWD mastoidectomy with type III tympanoplasty can be performed for prevention of disease recurrence or progression without fear for impairing hearing.

\section{REFERENCES}

1. Merchant SN, McKenna MJ, Rosowski JJ. Current status and future challenges of tympanoplasty. Eur Arch Otorhinolaryngol $1998 ; 255: 221-228$.

2. Sadé J. Introduction. In: Sade J (ed) Cholesteatoma and mastoid surgery 1982;1-3.

3. Adhikari P, Sinha BK, Pokhrel NR, Kharel B, Aryal R, Ma J. Prevalence of chronic suppurative otitis media in school children of kathmandu district. Journal of institute of medicine 2007;29(3):1012

4. Merchant SN, Rosowski JJ. Auditory physiology.GlassockShambough Surgery of the Ear, 5th ed. Elsevier India: New Delhi; 2003. p. 64-78.

5. American Academy of Otolaryngology-Head Neck Surgery Foundation, Inc. Committee on Hearing and Equilibrium guidelines for the evaluation of results of treatment of conductive hearing loss. Otolaryngol Head Neck Surg 1995;113:186-7.

6. De Corso E, Marchese MR, Sergi B, Rigante M, Paludetti G. Role of ossiculoplasty in canal wall down tympanoplasty for middle-ear cholesteatoma: hearing results. J Laryngol Otol 2007 Apr;121(4):324-8. Epub 2006 Nov 24.

7. Dawes PJd. Myringostapediopexy: surgical expectation. J Laryngol Otol 2003 Mar;117:182-185.

8. Kyrodimos E, Sismanis A, Santos D. Type III cartilage "shield” tympanoplasty: an effective procedure for hearing improvement. Otolaryngol Head Neck Surg 2007;136(6):982-5.

9. Merchant SN, McKenna MJ, Mehta RP, et al. Middle ear mechanics of type III tympanoplasty (stapes columella): II clinical studies. Otol Neurotol 2003;24(2):186 -94.

10. Cheang PP, Kim D, Rockley TJ. Myringostapediopexy and myringolenticulopexy in mastoid surgery. J Laryngol Otol 2008;17(3):1-5.

11. Mikaelian D. Perichondrial cartilage island graft in one stage tympano-ossiculoplasty. Laryngoscope1986;96:237-8.

12. Moustafa HM, Khalifa MA. Tympano-cartilago-stapediopexy: a method to improve hearing in open technique tympanoplasty. J Laryngol Otol 1990;104:942-4. 